\title{
Resistance phenotype and molecular epidemiology of Carbapenem-resistant Klebsiella pneumoniae isolates in Shanghai
}

\section{Wenxia Zhang}

Shanghai shuguang hospital

Hongyou Chen

Shanghai Municipal Center for Disease Control and Prevention

Chen Chen

shanghai shuguang hospital

Junhao Chen

shanghai shuguang hospital

Lingxia Li

shagnhai shuguang hospital

\section{Min Chen}

Shanghai Municipal Center for Disease Control and Prevention

Jue Zhang ( $\nabla$ zhangjue425@shutcm.edu.cn )

shanghai shuguang hospital

\section{Research}

Keywords: Klebsiella pneumoniae, KPC, genetic relatedness , MLST, PFGE

Posted Date: May 15th, 2020

DOl: https://doi.org/10.21203/rs.3.rs-22213/v1

License: (c) (i) This work is licensed under a Creative Commons Attribution 4.0 International License. Read Full License

Version of Record: A version of this preprint was published at Microbial Drug Resistance on July 23rd, 2021. See the published version at https://doi.org/10.1089/mdr.2020.0390. 


\section{Abstract}

Background: The emergence and wide global spread of carbapenem-resistant Klebsiella pneumoniae (CRKP) isolates are of great concern, and the aim of this study was to investigate drug resistance, molecular epidemiology, and genetic relationship of CRKP isolates from patients in Shanghai, China.

Methods: A retrospective study was conducted from April 2018 to July 2019, and a total of 133 CRKP isolates were collected. Antimicrobial susceptibility was determined by VITEK-2 automated microbiology analyzer platform (bioMérieux, France) and the broth microdilution method. Polymerase chain reaction (PCR) assays were used to investigate the presence of drug resistance genes. A modified carbapenem inactivation method ( $\mathrm{mCIM}$ ) was performed to detect carbapenemases. Multilocus sequence typing (MLST) and pulsed-field gel electrophoresis (PFGE) were conducted for genetic relatedness of $50 \mathrm{CRKP}$ isolates selected.

Results: Among 670 isolates of K. pneumoniae, 133 (19.85\%) strains were identified as carbapenemresistant K. pneumoniae (CRKP), of which, 76.69\% (102/133) strains were isolated from ICUs. All the 133 CRKP isolates were found to be carbapenemase-producers and harbor blaKPC-2 gene. No other carbapenemase genes of blaNDM, blaOXA-48, blaVIM, and blaIMP were detected. Furthermore, $\beta$ lactamase genes of blaSHV, blaCTX, and blaTEM were the most common resistance-associated genes among these KPC-2 producing isolates. All the 133 CRKP strains displayed more than $95 \%$ of resistance to cephalosporins and carbapenems, except for gentamicin, Trimethoprim-sulfamethoxazole, amikacin, tigecycline and colistin. The most common sequence type was ST11, accounting for $90.0 \%$ of the 50 CRKP selected, followed by ST15 (10\%). PFGE analysis clustered the 50 KPC-2-producing isolates into seven (A-G) distinct clonal clusters at $85 \%$ cut off. Of which, cluster $A$ and $G$ were the two major clusters, accounting for the majority of the strains collected in emergency ICU and neurosurgical ICU. And all the strains of cluster D and E were collected in cardiothoracic surgery ICU, expect for one strain collected in one outpatient.

Conclusion: The KPC-2-producing K.pneumoniae belonged to ST11 was widely disseminated in ICUs, and active and effective surveillance of infection control strategies was initiated to limit the spread of CRKP strains.

\section{Introduction}

Klebsiella pneumoniae is a common nosocomial infection pathogen in hospitals, especially the infection caused by carbapenem-resistant $K$. pneumoniae (CRKP) clinical isolates has spread widely throughout the world ${ }^{[1-4]}$. According to the results of 12-year bacterial resistance monitoring from China Antimicrobial Surveillance Network (CHINET), the significant rising resistance trend of $K$. pneumoniae to impenem or meropenem, from $3.0 \%$ and $2.9 \%$ in 2005 to $25.0 \%$ and $26.3 \%$ in $2018^{[5]}$. Since the CRKP strain is usually resistant to the most antimicrobials commonly used in clinical practice, few therapeutic 
options are remaining. Therefore, treatment of CRKP infections are extremely challenging due to its high levels of morbidity and mortality ${ }^{[6]}$, especially for patients in intensive care unit (ICU).

The main cause of carbapenem resistance is the production of carbapenemase such as $K$. pneumoniae carbapenemases (KPCs), New Delhi metallo- $\beta$-lactamase (NDM) and oxacillinase type 48 (OXA-48). In China, KPC-producing K. pneumoniae strains were the most predominant in adults, while the NDMproducing strains were mainly isolated in children ${ }^{[7]}$. The both carbapenemase genes are predominantly plasmid encoded, which facilitated the highly transmission across multiple enterobacterial species. Therefore, understanding the resistance phenotype and molecular epidemiology of CRKP isolates is essential to control the wide spread of these strains and promoting the selection and efficacy of empirical therapy.

Thus, a retrospective study in our hospital from April 2018 to July 2019 focusing on the molecular epidemiology of CRKP strains was conducted to provide reference for clinical anti-infection treatment and the control of nosocomial infection.

\section{Methods}

\section{Bacterial isolates}

From April 2018 to July 2019, a total of 133 carbapenem-resistant K. pneumoniae isolates were consecutively collected in Shuguang Hospital, Affiliated to Shanghai University of Traditional Chinese Medicine, Shanghai, China. These 133 strains represented $19.85 \%$ of all isolates of 670 non-duplicated $K$. pneumoniae cultured form patients during the study period. All isolates were identified by the VITEK-2 system.

Antimicrobial susceptibility testing and phenotypic analysis

Antimicrobial susceptibility testing was performed by VITEK-2 system and broth microdilution method, and the results were interpreted according to 2018 Clinical and Laboratory Standards Institute(CLSI) guidelines ${ }^{[8]}$ except for colistin and tigecycline, which according to the European Committee on Antimicrobial Susceptibility Testing (EUCAST) ${ }^{[9]}$ interpretive criteria(http://www.eucast.org/). Escherichia coli ATCC 25922 was used as the quality control for antimicrobial susceptibility testing. The definition of a CRKP strain is resistant to imipenem or meropenem. A modified carbapenem inactivation method ( $\mathrm{mCIM}$ ) was performed to detect carbapenemases, as described previously ${ }^{[10]}$.

Detection of antimicrobial resistance genes

Polymerase chain reaction (PCR) assays were performed as described previously to investigate the presence of drug resistance genes, including carbapenemase-encoding genes (blaKPC, blaNDM, blaOXA - 48, blaVIM, and blaIMP), AmpC genes (DHA, FOX, $A A C, E B C$, and $C I T)$ and extended-spectrum $\beta$ lactamase (ESBL)-encoding genes (blaCTX, blaSHV, and blaTEM) ${ }^{[11-14]}$. Pulsed-field gel electrophoresis (PFGE) 
Genetic relatedness among CRKP strains was performed using PFGE method according to the protocol outlined in the PulseNet for Enterobacteriaceae. In brief, the whole genomic DNA was prepared by agarose embedding method, followed by Xbal digestion with the Bio-Rad CHEF Mapper electrophoresis system. Salmonella serotype Braenderup strain $\mathrm{H} 9812$ was used as a control strain and molecular marker. The PFGE banding pattern was clustered by BioNumerics software using the Dice Similarity coefficient.

Multilocus sequence typing (MLST)

MLST was performed by amplifying the seven conserved $K$. pneumoniae housekeeping genes, including gapA, infB, $m d h, p g i, p h o E, r p o B$, and tonB. Alleles and sequence types (STs) were assigned according to the protocol available on the Institute Pasteur MLST database website (http://www.pasteur.fr/recherche/genopole/PF8/mlst/Kpneumoniae.html).

\section{Results}

\section{Clinical characteristics}

Among 670 isolates of $K$. pneumoniae, 133 (19.85\%) strains were identified as carbapenem-resistant $K$. pneumoniae (CRKP). Most CRKP isolates were detected in the intensive care unit $(76.69 \%, 102 / 133)$, including emergency ICU, neurosurgical ICU, cardiothoracic surgery ICU, neurological ICU and respiratory ICU, followed by neurosurgery wards $(8.27 \%, 11 / 133)$ and gastroenterology wards $(6.77 \%, 9 / 133)$. And the majority of CRKP strains were isolated from sputum $(60.90 \%, 81 / 133)$, followed by bile $(9.02 \%, 12 / 133)$, urine $(9.02 \%, 12 / 133)$, blood $(6.02 \%, 8 / 133)$, and aseptic body fluids samples $(5.26 \%, 7 / 133)$ such as cerebrospinal fluid, BALF and drainage.

\section{Antimicrobial Susceptibility}

The antimicrobial resistance rates of the 133 CRKP isolates were shown in Table 1 . They displayed more than $95 \%$ of resistance to cephalosporins and carbapenems, apart from gentamicin, Trimethoprimsulfamethoxazole, amikacin, tigecycline and Colistin. 
Table 1

Resistance to antimicrobial agents of 133 CRKP isolates

\begin{tabular}{|lccl|}
\hline Antimicrobial Agent & R(\%) & I(\%) & S(\%) \\
\hline Cefazolin & 100.0 & 0.0 & 0.0 \\
\hline Cefuroxime & 100.0 & 0.0 & 0.0 \\
\hline Ceftazidime & 100.0 & 0.0 & 0.0 \\
\hline Cefotaxime & 100.0 & 0.0 & 0.0 \\
\hline Cefatriaxone & 100.0 & 0.0 & 0.0 \\
\hline Cefepime & 100.0 & 0.0 & 0.0 \\
\hline Piperacillin-tazobactam & 100.0 & 0.0 & 0.0 \\
\hline Ampicillin-sulbactam & 100.0 & 0.0 & 0.0 \\
\hline Imipenem & 100.0 & 0.0 & 0.0 \\
\hline Meropenem & 99.2 & 0.8 & 0.0 \\
\hline Cefoperazone-sulbactam & 99.2 & 0.0 & 0.8 \\
\hline Levofloxacin & 98.5 & 0.8 & 0.8 \\
\hline Gentamicin & 75.2 & 2.3 & 22.6 \\
\hline Trimethoprim-sulfamethoxazole & 72.9 & 0.0 & 27.1 \\
\hline Ampicillin & 30.1 & 0.8 & 69.2 \\
\hline Tegafycline & 3.0 & 0.0 & 97.0 \\
\hline Colistin & 2.3 & 0.0 & 97.7 \\
\hline R Resistant, I Intermediary, S Susceptible & & \\
\hline
\end{tabular}

\section{Molecular Characteristics of CRKP Isolates}

All 133 CRKP were found to be carbapenemase-producers determined by modified carbapenem inactivation method ( $\mathrm{mCIM}$ ). And the carbapenemase gene of blaKPC-2 was detected in all these strains, which was in accordance with the phenotypic results. No other carbapenemase genes of blaNDM, blaOXA - 48, blaVIM, and blaIMP were detected. In addition to blaKPC-2 gene, $\beta$-lactamase genes of blaSHV, blaCTX, and blaTEM were identified in $88.7 \%$ (118/133), 55.6\% (74/133) and $77.4 \%(103 / 133)$ isolates, respectively. While, only 4 strains were positive for gene $D H A$, encoding plasmid-mediated AmpCs.

Of the 133 blaKPC-2-producing Klebsiella pneumoniae isolates, PFGE and MLST analysis were further performed on a subset of 50 isolates. Isolates were selected according to the respective proportions, 
specimen types and isolated intervals of these blaKPC-2-positive $K$. pneumoniae strains per ward, to provide a representative image of the different wards.

MLST analysis showed that the most common sequence type was ST11, accounting for $90.0 \%$ of the 50 CRKP selected, followed by ST15 (10.0\%). PFGE analysis revealed seven distinct clusters and related subclusters of the 50 blaKPC-2-positive isolates with $85 \%$ and $95 \%$ cutoff genetic similarity, relatively, as shown in Fig. 1. All the tested isolates of PFGE clusters A-F were belonged to MLST type ST11 and only five isolates of PFGE cluster $\mathrm{G}$ were belonged to MLST type ST15. Furthermore, cluster A and G appeared dominantly represented, comprising $16(32 \%)$ isolates and 22(44\%) isolates, respectively. Of which, cluster A accounted for the majority of the strains collected in emergency ICU and cluster G accounted for isolates mostly in neurosurgical ICU and emergency ICU. And all the strains of cluster D and E were collected in cardiothoracic surgery ICU, expect for one strain collected in one outpatient.

\section{Discussion}

Intensive Care Unit (ICU) is the main department of nosocomial infections and has been considered as a factory for manufacturing, disseminating, and enhancing antimicrobial resistance ${ }^{[15]}$. The increasing incidence of antimicrobial resistance of isolates from ICU is becoming more and more serious due to the complicated conditions such as extremely vulnerable critically ill patients with many basic diseases, use of invasive operations, relatively long hospitalization time and excessive use of generally broad-spectrum antimicrobials, which bring difficulties to clinical anti-infective treatment ${ }^{[16,17]}$. Meanwhile, the infections caused by CRKP are significantly increasing over time ${ }^{[18]}$, which pose a challenge to clinical treatment and negative impact on patient outcome due to the limited availability of clinically antimicrobials.

In our study, among 133 CRKP strains, 102 were isolated in the intensive care unit, accounting for $76.69 \%$, showing the high prevalence of patients, which was consistence with previous studies ${ }^{[19,20]}$. Additional, the isolation rate of CRKP in sputum was the highest (60.90\%), which might be due to the heavy use of ventilator reported as the major risk factor of CRKP infections in ICUs.

All the 133 CRKP isolates were found to be carbapenemase-producers and harbor bla KPC-2 gene, which accounted for the most frequent mechanism for resistance to carbapenems in K. pneumoniae. In China, KPC-producing-K.pneumoniae was the most prevailing and reported as the predominant outbreak strain $^{[21,22]}$. Fortunately, none multi-carbapenemase-producing K.pneumoniae was observed in this study. However, further analysis revealed the high prevalence of the combination of three $\beta$-lactamases genes of blaSHV, blaCTX, and blaTEM among these KPC-2 producing strains. The co-existence of resistant determinants with the combination of carbapenemase and two or more $\beta$-lactamases leaded to the multidrug resistance.

As a result, all the isolates displayed a multi-resistant phenotype, with all resistant to cephalosporins and carbapenems, such as cefazolin, cefuroxime, cefatriaxone, ceftazidime, cefotaxime, cefepime, piperacillin-tazobactam, ampicillin-sulbactam, and imipenem. Although only $3.0 \%$ and $2.3 \%$ isolates 
showed resistance to tegafycline and Colistin, respectively, the two antimicrobials had the disadvantages of low plasma concentration and significant toxicity. Thus, treatment of infections caused by these multiresistant isolates are extremely challenging due to its limited antimicrobials and high levels of morbidity and mortality.

According to MLST typing, ST11 was the most dominant sequence type of the 50 CRKP isolates, accounting for $90.0 \%$, which was consistent with previous studies in China ${ }^{[15,19]}$. In addition, ST11 clone is the single locus variant of ST258, and that they belong to the same clonal members of CG258[23], which has contributed largely to the global dissemination of KPC carbapenemase over the past 20 years ${ }^{[24,25]}$. The difference is that the ST258 clone is prevailing in North America and Europe, while the ST11 clone is frequently isolated in Asia ${ }^{[25]}$. Moreover, ST11 clone showed multidrug-resistant phenotype with high carriage rate of virulence genes. These virulence factors are conducive to binding, biofilm formation, colonization, and anti-phagocytosis, enhancing environmental survival and, which can successfully demonstrate their persistence and quick expansion across the world ${ }^{[26,27]}$.

In addition to ST11, five ST15 KPC-2-producing K. pneumoniae were isolated in patients in neurosurgical ICU, gastroenterology ward, emergency ICU and outpatient, relatively. It was worth noting that $K$. pneumoniae isolates of ST15 was widely disseminated in Europe and repeatedly reported to spread carbapenemase genes such as NDM-1 in North African ${ }^{[28]}$ and in Greece ${ }^{[29]}$ and OXA-48 in Spain ${ }^{[30]}$. However, ST15 is gradually detected in China ${ }^{[31,32]}$. Moreover, in our study one ST15 strain was isolated from one outpatient who had not been hospitalized or received health care services in the previous 3 months, suggesting that ST15 is a potential risk clone to be a community onset which deserves routine surveillance to prevent outbreak.

MLST is appropriate for genotyping to further provide data not only for local epidemiological investigations but also for global and evolutionary studies. However, since MLST only involve seven housekeeping genes, it is less discriminating than PFGE to analyze outbreaks in different wards. In our study, all the ST11 isolates were divided into A-G seven different PFGE clusters and related sub-clusters. Of which, sub-cluster A1 and G1 appeared the two most predominant clones with $>95 \%$ similarity of PFGE patterns. And the sub-cluster A1 consisted of all the isolates collected in emergency ICU, expect for two isolates in neurosurgical, and sub-cluster $\mathrm{G} 1$ in both neurosurgical ICU and emergency ICU, suggesting that the two prevalent clones were transmitted in the two ICUs. In addition, there were two isolates assigned to cluster $\mathrm{B}$ and one isolate belonged to cluster $\mathrm{F}$ were also detected in emergency ICU, which suggested us that emergency ICU was one of the most high-risk wards and might be the focal point of active monitoring for infection control.

Finally, the six isolates belonged to cluster $\mathrm{D}$ and $\mathrm{E}$ were observed just in cardiothoracic surgery ICU, except one in an outpatient, which might had different genetic backgrounds from the isolates in neurosurgical ICU and emergency ICU. It was worth noting that the CRKP isolates assigned to cluster A, B, $\mathrm{C}, \mathrm{E}$ and $\mathrm{G}$ all had the ability to cause blood infection, which deserved our attention. 
In conclusion, our study revealed that ICUs were the main wards for rapid and wide spread of CRKP and clonal dissemination and outbreak of KPC-2-producing CRKP belonged to ST11 was observed in ICUs, especially in emergency ICU in our hospital. Hence,routine surveillance and effective infection control strategies are urgently warranted on these high-risk international strains to prevent outbreak and nosocomial-acquired infection.

\section{Abbreviations}

CRKP

carbapenem-resistant Klebsiella pneumoniae

PCR

Polymerase chain reaction

mCIM

modified carbapenem inactivation method

MLST

Multilocus sequence typing

PFGE

pulsed-field gel electrophoresis

ICU

Intensive Care Unit

\section{Declarations}

\section{Ethics approval and consent to participate:}

This study was approved by Shuguang hospital.

\section{Consent for publication:}

Not applicable.

\section{Availability of data and material:}

The datasets used and/or analysed during the current study are available from the corresponding author on reasonable request.

\section{Competing interests:}

We have no conflicts of interest to declare. 


\section{Funding:}

No funding.

\section{Authors' contributions:}

All authors read and approved the final version of the manuscript.

\section{Acknowledgements:}

We thank all the laboratories for contributing data to this analysis.

\section{References}

1. B W, F P, C W, et al. Molecular epidemiology of Carbapenem-resistant Klebsiella pneumoniae in a paediatric hospital in China. International journal of infectious diseases: IJID : official publication of the International Society for Infectious Diseases. 2020.

2. P N, G C, T N. The real threat of Klebsiella pneumoniae carbapenemase-producing bacteria. The Lancet Infectious diseases. 2009,9(4):228-236.

3. TB MC. D, V K, et al. EnterobacteralesMeropenem-Vaborbactam Activity against CarbapenemResistant Isolates Collected in U.S. Hospitals during 2016 to 2018. Antimicrobial agents and chemotherapy. 2020,64(2).

4. Liu J, Yu J, Chen F, et al. Emergence and establishment of KPC-2-producing ST11 Klebsiella pneumoniae in a general hospital in Shanghai, China. Eur J Clin Microbiol Infect Dis. 2018;37(2):293-9.

5. F H, Y G, Y Y, et al. Resistance reported from China antimicrobial surveillance network (CHINET) in 2018. European journal of clinical microbiology \& infectious diseases: official publication of the European Society of Clinical Microbiology. 2019,38(12):2275-2281.

6. Hauck C, Cober E, Richter SS, et al. Spectrum of excess mortality due to carbapenem-resistant Klebsiella pneumoniae infections. Clin Microbiol Infect. 2016;22(6):513-9.

7. Tian D, Pan F, Wang C, et al. Resistance phenotype and clinical molecular epidemiology of carbapenem-resistant Klebsiella pneumoniae among pediatric patients in Shanghai. Infect Drug Resist. 2018;11:1935-43.

8. Clinical and Laboratory Standards Institute (CLSI). Performance Standards for Antimicrobial Susceptibility Testing twentieth informational supplement. Wayne: CLSI; 2018.

9. The European Committee on Antimicrobial Susceptibility Testing. Breakpoint tables for interpretation of MICs and zone diameters. European Committee on Antimicrobial Susceptibility Testing (EUCAST). 2018. 
10. Pierce VM, Simner PJ, Lonsway DR, et al. Modified Carbapenem Inactivation Method for Phenotypic Detection of Carbapenemase Production among Enterobacteriaceae. J Clin Microbiol. 2017;55(8):2321-33.

11. Wang P, Chen S, Guo Y, et al. Occurrence of false positive results for the detection of carbapenemases in carbapenemase-negative Escherichia coli and Klebsiella pneumoniae isolates. PLoS One. 2011;6(10):e26356.

12. Revathi G, Siu LK, Lu PL, et al. First report of NDM-1-producing Acinetobacter baumannii in East Africa. Int J Infect Dis. 2013;17(12):e1255-8.

13. Poirel L, Walsh TR, Cuvillier V, et al. Multiplex PCR for detection of acquired carbapenemase genes. Diagn Microbiol Infect Dis. 2011;70(1):119-23.

14. Perez-Perez FJ, Hanson ND. Detection of plasmid-mediated AmpC beta-lactamase genes in clinical isolates by using multiplex PCR. J Clin Microbiol. 2002;40(6):2153-62.

15. Q Y. X J, M Z, et al. Klebsiella pneumoniaeEmergence of ST11-K47 and ST11-K64 hypervirulent carbapenem-resistant in bacterial liver abscesses from China: a molecular, biological, and epidemiological study. Emerging microbes infections. 2020;9(1):320-31.

16. C YLHS. Z, et al. Klebsiella pneumoniaeCarbapenem-Resistant Infections among ICU Admission Patients in Central China: Prevalence and Prediction Model. BioMed research international. 2019;2019:9767313.

17. WM Z ZY, HY Z. Risk factors for carbapenem-resistant Klebsiella pneumoniae infection relative to two types of control patients: a systematic review and meta-analysis. Antimicrobial resistance infection control. 2020;9(1):23.

18. Wang C, Yuan Z, Huang W, et al. Epidemiologic analysis and control strategy of Klebsiella pneumoniae infection in intensive care units in a teaching hospital of People's Republic of China. Infect Drug Resist. 2019;12:391-8.

19. X Q. S W, M H, et al. The Colonization of Carbapenem-Resistant Klebsiella pneumoniae:

Epidemiology, Resistance Mechanisms, and Risk Factors in Patients Admitted to Intensive Care Units in China. The Journal of infectious diseases. 2020;221:206-14.

20. F Y. L H, Q Z, et al. Dissemination of Klebsiella pneumoniae ST11 isolates with carbapenem resistance in integrated and emergency intensive care units in a Chinese tertiary hospital. Journal of medical microbiology. 2019;68(6):882-9.

21. S S, C L, Y Z, et al. Klebsiella pneumoniaeOutbreak of KPC-2-Producing ST76 Isolates in an Intensive Care Unit and Neurosurgery Unit. Microbial drug resistance (Larchmont, NY). 2020.

22. X C, G H. H X, et al. Klebsiella PneumoniaeGenomic Analysis Of A KPC-2-Producing ST11 Outbreak From A Teaching Hospital In Shandong Province, China. Infection and drug resistance. 2019,12:2961-2969.

23. L C, B M, KD C, et al. Carbapenemase-producing Klebsiella pneumoniae: molecular and genetic decoding. Trends in microbiology. 2014,22(12):686-696. 
24. D G. N D, Z Z, et al. A fatal outbreak of ST11 carbapenem-resistant hypervirulent Klebsiella pneumoniae in a Chinese hospital: a molecular epidemiological study. The Lancet Infectious diseases. 2018;18(1):37-46.

25. LS M-P LP. RA B, et al. Clinical epidemiology of the global expansion of Klebsiella pneumoniae carbapenemases. The Lancet Infectious diseases. 2013;13(9):785-96.

26. K Z. T X, S D, et al. Novel Subclone of Carbapenem-Resistant Klebsiella pneumoniae Sequence Type 11 with Enhanced Virulence and Transmissibility, China. Emerg Infect Dis. 2020;26(2):289-97.

27. LN A, GG LV. G, et al. Expansion and evolution of a virulent, extensively drug-resistant (polymyxin Bresistant), QnrS1-, CTX-M-2-, and KPC-2-producing Klebsiella pneumoniae ST11 international highrisk clone. J Clin Microbiol. 2014;52(7):2530-5.

28. $L V, L P, P ~ N$, et al. Complete sequencing of an IncH plasmid carrying the blaNDM-1, blaCTX-M-15 and qnrB1 genes. The Journal of antimicrobial chemotherapy. 2012,67(7):1645-1650.

29. K LP. G, N S, et al. Emergence of NDM-1-producing Klebsiella pneumoniae in Greece: evidence of a widespread clonal outbreak. J Antimicrob Chemother. 2019;74(8):2197-202.

30. S F-R AMJGG, et al. Dissemination and clinical implications of multidrug-resistant Klebsiella pneumoniae isolates producing OXA-48 in a Spanish hospital. J Hosp Infect. 2017;96(2):116-22.

31. X L, W M. Q Q, et al. Nosocomial spread of OXA-232-producing Klebsiella pneumoniae ST15 in a teaching hospital, Shanghai, China. BMC microbiology. 2019;19(1):235.

32. C M. J M, A B, et al. Klebsiella pneumoniae causing urinary tract infections in companion animals and humans: population structure, antimicrobial resistance and virulence genes. J Antimicrob Chemother. 2019;74(3):594-602.

\section{Figures}




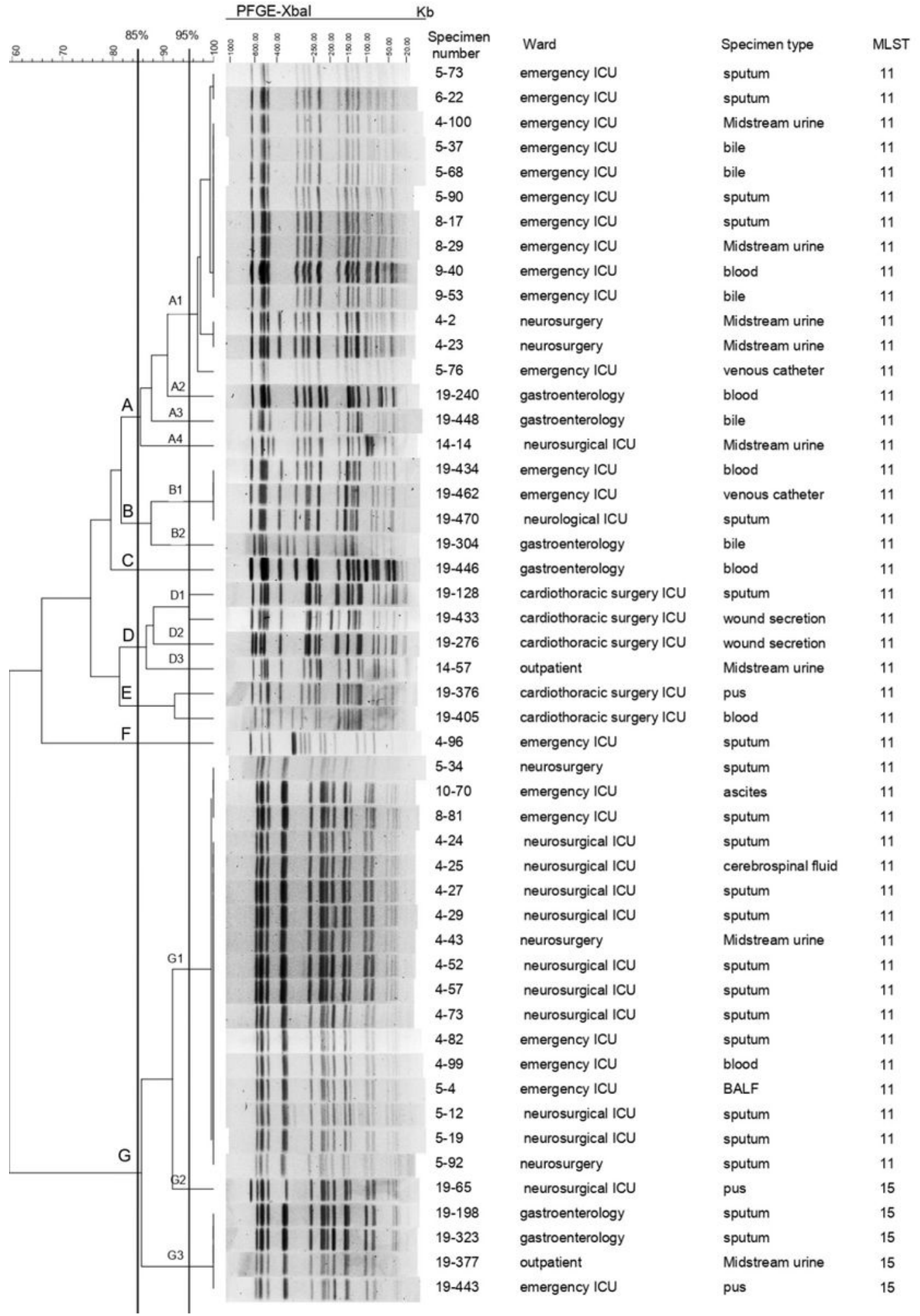

\section{Figure 1}

Relationships of the 50 KPC-2-producing K. pneumoniae isolates based on Pulsed Field Gel Electrophoresis (PFGE) and MLST 